\title{
Cosmological hydrogen recombination: Ly $n$ line feedback and continuum escape
}

\author{
J. Chluba ${ }^{1}$ and R. A. Sunyaev ${ }^{1,2}$ \\ 1 Max-Planck-Institut für Astrophysik, Karl-Schwarzschild-Str. 1, 85741 Garching bei München, Germany \\ e-mail: jchluba@mpa-garching.mpg.de \\ 2 Space Research Institute, Russian Academy of Sciences, Profsoyuznaya 84/32, 117997 Moscow, Russia
}

Received 20 February 2007 / Accepted 16 September 2007

\begin{abstract}
We compute the corrections to the cosmological hydrogen recombination history due to delayed feedback of Lyman-series photons and the escape in the Lyman-continuum. The former process is expected to slightly delay recombination, while the latter should allow the medium to recombine a bit faster. It is shown that the subsequent feedback of released Lyman- $n$ photons on the lower lying Lyman- $(n-1)$ transitions yields a maximal correction of $\Delta N_{\mathrm{e}} / N_{\mathrm{e}} \sim 0.22 \%$ at $z \sim 1050$. Including only Lyman- $\beta$ feedback onto the Lyman- $\alpha$ transition, accounts for most of the effect. We find corrections to the cosmic microwave background TT and EE power spectra with typical peak to peak amplitude $\left|\Delta C_{l}^{T T} / C_{l}^{T T}\right| \sim 0.15 \%$ and $\left|\Delta C_{l}^{E E} / C_{l}^{E E}\right| \sim 0.36 \%$ at $l \lesssim 3000$. The escape in the Lymancontinuum and feedback of Lyman- $\alpha$ photons on the photoionization rate of the second shell lead to modifications of the ionization history which are very small (less than $\left|\Delta N_{\mathrm{e}} / N_{\mathrm{e}}\right| \sim$ few $\times 10^{-6}$ ).
\end{abstract}

Key words. cosmic microwave background - cosmology: miscellaneous

\section{Introduction}

The tremendous advances in observations of the cosmic microwave background (CMB) temperature and polarization angular fluctuations (e.g. Page et al. 2006; Hinshaw et al. 2006) and the prospects with the PLANCK Surveyor ${ }^{1}$ have motivated several groups to re-examine the problem of cosmological hydrogen recombination, with the aim of identifying previously neglected physical processes which could affect the ionization history of the Universe at the level of $\gtrsim 0.1 \%$, and may lead to modifications of the cosmological hydrogen recombination spectrum (Dubrovich 1975; Beigman \& Sunyaev 1978; Rybicki \& dell'Antonio 1993; Dubrovich \& Stolyarov 1995; Burgin 2003; Dubrovich \& Shakhvorostova 2004; Kholupenko et al. 2005; Wong et al. 2006; Rubiño-Martín et al. 2006; Chluba \& Sunyaev 2006a; Chluba et al. 2007), which could become observable in the future. For example, effects connected with the two-photon transitions from high $\mathrm{s}$ and d-states to the ground state (Dubrovich \& Grachev 2005; Wong \& Scott 2007; Chluba \& Sunyaev 2007), the induced 2s-two-photon decay (Chluba \& Sunyaev 2006b), the increase in the 2s-two-photon absorption rate due to the large Lyman- $\alpha$ spectral distortion (Kholupenko \& Ivanchik 2006), and details in the evolution of the populations of the angular momentum sub-states (Rubiño-Martín et al. 2006; Chluba et al. 2007) were discussed. All these studies show that sub-percent-level corrections to the ionization history do exist, which in principle could bias the values of the key cosmological parameters (Lewis et al. 2006). It is clear, that when reaching percent-level accuracy in the determination of the key cosmological parameters or when considering signatures from inflation, e.g. the possibility of a running spectral index, accurate

\footnotetext{
1 wWw.rssd.esa.int/Planck
}

theoretical predictions of the ionization history are required. Here we examine the effects due to feedback of hydrogen Lyman-series photons and the escape of photons in the Lymancontinuum on the ionization history during the epoch of hydrogen recombination, and the impact of this process on the Lyman$\alpha$ distortion of the CMB blackbody spectrum.

The strongest distortions of the CMB spectrum arising during the epoch of hydrogen recombination are due to the Lyman- $\alpha$ transition and the 2s two-photon decay (Zeldovich et al. 1968; Peebles 1968). The feedback of these excess photons on the photoionization rates of the second shell has been considered by Seager et al. (1999, 2000), with no significant changes for the number density of free electrons, $N_{\mathrm{e}}$. We confirm this statement for the feedback from Lyman- $\alpha$ photons and find a maximal correction of $\Delta N_{\mathrm{e}} / N_{\mathrm{e}} \sim 10^{-6}$ at $z \sim 500$, the redshift which roughly corresponds to the time, when the maximum of the CMB spectral distortion due to the Lyman- $\alpha$ transition is reaching the Balmer-continuum frequency.

However, it has been shown by Kholupenko \& Ivanchik (2006) that the huge excess of photons in the Wien-tail of the CMB due to the Lyman- $\alpha$ distortion leads to an increase of the $1 \mathrm{~s} \rightarrow 2 \mathrm{~s}$ two-photon absorption rate, which delays recombination and introduces corrections to the ionization history at the percent-level. Similarly, one expects some feedback of escaping Lyman-series photons on lower lying Lymantransitions and eventually the Lyman- $\alpha$ transition. Recently, this process was also considered by Switzer \& Hirata (2007) for both helium and hydrogen recombination, using an iterative approach. Here we directly compute the escaping Lyman- $n$ radiation within the Sobolev approximation (e.g. see Rubiño-Martín et al. 2006; Chluba et al. 2007) and include the feedback on the Lyman- $(n-1)$ transition at all times by evaluating the distorted 
Table 1. Appearance of the first few Lyman lines for the computations not including any feedback. In particular we give the approximate redshift, $z_{\max }$, at which $\Delta I_{v}\left(z_{\mathrm{em}}\right)$ is maximal (see Fig. 1), and the redshift at which the peak of the line reaches the next lower Lyman resonance, $z_{\mathrm{f}} \approx z_{\max } \nu_{\mathrm{Ly}(n-1)} / \nu_{\mathrm{Ly} n}$, where $v_{\mathrm{Lyk}}$ is the resonance frequency of the Lyman- $k$ transition. Also we give the approximate total number of escaping photons per hydrogen nucleus for each transition.

\begin{tabular}{lccccc}
\hline \hline Line & $n$ & $v_{\mathrm{Ly} n}[\mathrm{~Hz}]$ & $z_{\max }$ & $z_{\mathrm{f}}$ & $N_{\gamma} / N_{\mathrm{H}}$ \\
\hline Lyman- $\alpha$ & 2 & $2.4674 \times 10^{15}$ & 1400 & - & $4.3 \times 10^{-1}$ \\
Lyman- $\beta$ & 3 & $2.9243 \times 10^{15}$ & 1450 & 1223 & $1.8 \times 10^{-3}$ \\
Lyman- $\gamma$ & 4 & $3.0842 \times 10^{15}$ & 1461 & 1385 & $2.9 \times 10^{-4}$ \\
Lyman- $\delta$ & 5 & $3.1583 \times 10^{15}$ & 1464 & 1430 & $1.3 \times 10^{-4}$ \\
Lyman- $\epsilon$ & 6 & $3.1985 \times 10^{15}$ & 1467 & 1449 & $7.9 \times 10^{-5}$ \\
\hline
\end{tabular}

spectrum at the corresponding frequency. In addition, we assume that due to the huge optical depth in all the Lyman-lines, at the end only the Lyman- $\alpha$ line remains and all the Lyman- $n$ lines will be completely re-processed by the closest lower lying Lyman- $(n-1)$ transition. Therefore, feedback always only works as $\operatorname{Ly} n \rightarrow \operatorname{Ly}(n-1)$. It is also important to ensure that the line is not producing any feedback on itself.

In addition, we estimate the escape probability for the Lyman-continuum and include the approximate net rate for direct recombinations into our multi-level code. As argued earlier (Zeldovich et al. 1968; Peebles 1968), we find that the modification of the ionization history due to this process is indeed completely negligible.

\section{Re-absorption of Lyman-series photons}

Although in computations with no feedback, the contributions of the $n \mathrm{p} \rightarrow 1 \mathrm{~s}$ transitions from shells with $n>2$ to the total Lyman-series are rather small (see Fig. 8 in Rubiño-Martín et al. 2006), in the Wien-tail of the CMB the intensity of these lines is significantly larger than the blackbody (see Fig. 1). Therefore one expects that after redshifting each Lyman-transition from $n>2$ will increase the absorption rate for the closest lower Lyman-series transition $\left(n^{\prime}=n-1 \geq 2\right)$ and thereby slightly delay hydrogen recombination. For example, the Lyman- $\beta$ transition peaks at $z_{\mathrm{em}} \sim 1450$ with contribution $\Delta I_{v} / B_{v} \sim 64 \%$ relative to the CMB blackbody spectrum $B_{v}$. These excess photons reach the central frequency of the Lyman- $\alpha$ transition at $z \sim 1223$ and increase the Lyman- $\alpha$ absorption rate, thereby delaying recombination (see Table 1). It is easy to estimate the total number of photons released in the Lyman- $\beta$ transition using Fig. 1. Its value is close to $\sim 0.18 \%$ of the total number of hydrogen nuclei (see Table 1 for the other transitions). At the time of maximal feedback $\left(z_{\mathrm{f}} \sim 1223\right)$ the number of free electrons is roughly $38 \%$ of the total number of hydrogen nuclei. Therefore the maximal effect one can expect for the feedbackinduced corrections to the ionization history from the Lyman- $\beta$ line is $\Delta N_{\mathrm{e}} / N_{\mathrm{e}} \lesssim 0.5 \%$.

In Fig. 2 we present the results for detailed computations of feedback-induced changes in the free electron fraction. Including only Lyman- $\beta$ feedback on the Lyman- $\alpha$ transition accounts for most of the effect, leading to a maximal difference of $\Delta N_{\mathrm{e}} / N_{\mathrm{e}} \sim 0.25 \%$ at $z \sim 1040$.

When including in addition the feedback of Lyman- $\gamma$ on Lyman- $\beta$, the maximal relative difference decreases to $\Delta N_{\mathrm{e}} / N_{\mathrm{e}} \sim$ $0.20 \%$, now peaking at $z \sim 1070$. This behavior is expected, since the Lyman- $\gamma$ photon reduces the transition rate in the Lyman- $\beta$ channel such that its feedback on the Lyman- $\alpha$ line
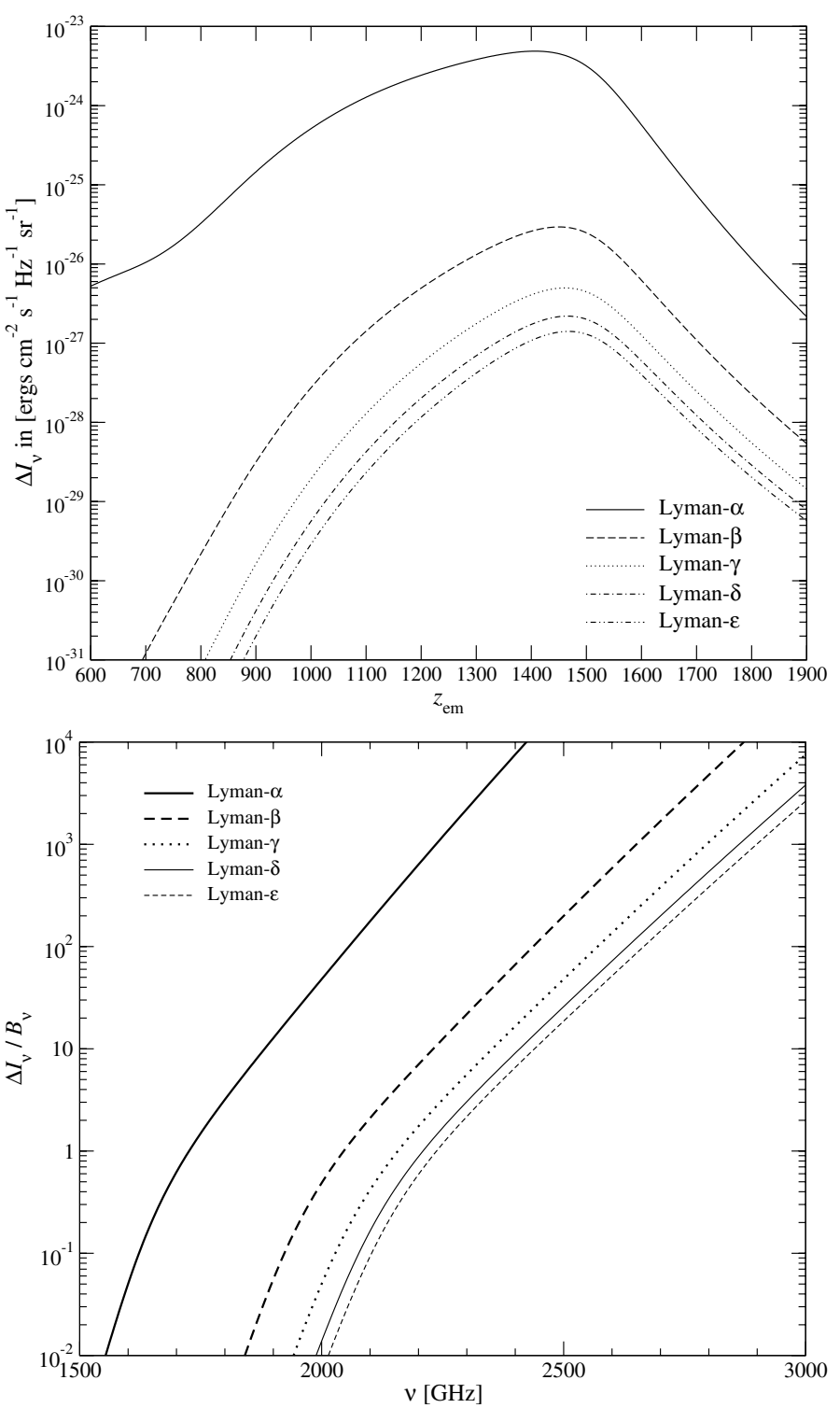

Fig. 1. Spectral distortions due to the first few Lyman-transitions at $z=0$. No feedback $\operatorname{Ly} n \rightarrow \operatorname{Ly}(n-1)$ has been included. Upper panel: as a function of the redshift of emission (see Eq. (1) in Rubiño-Martín et al. 2006). Lower panel: relative to the CMB blackbody as a function of observing frequency $v$.

should become slightly smaller. In addition, the number of electrons reaching the ground state via the Lyman- $\beta$ transition will be reduced, which very likely leads to the small increase of the correction at $z \gtrsim 1200$.

Subsequent inclusion of more feedback results in an alternating behavior of $\Delta N_{\mathrm{e}} / N_{\mathrm{e}}$. We found that for 30-shells, convergence is reached when including feedback from $n$ p-levels with $n \lesssim 15$. In Fig. 2 we also give the full result including all feedback within a 30-shell hydrogen atom. In addition we found that the correction barely depends on the total number of included shells. Therefore we expect that even for computations with up to 100 -shells the total feedback-induced relative difference will not change significantly.

Examining the final differences in the Lyman- $\alpha$ distortion and the feedback-induced modifications of the Lyman- $\beta$ and $\gamma$ lines before their absorption within the corresponding lower lying resonance (see Fig. 3) shows that the situation is a bit more complicated. Including only Lyman- $\beta$ feedback the amount of 


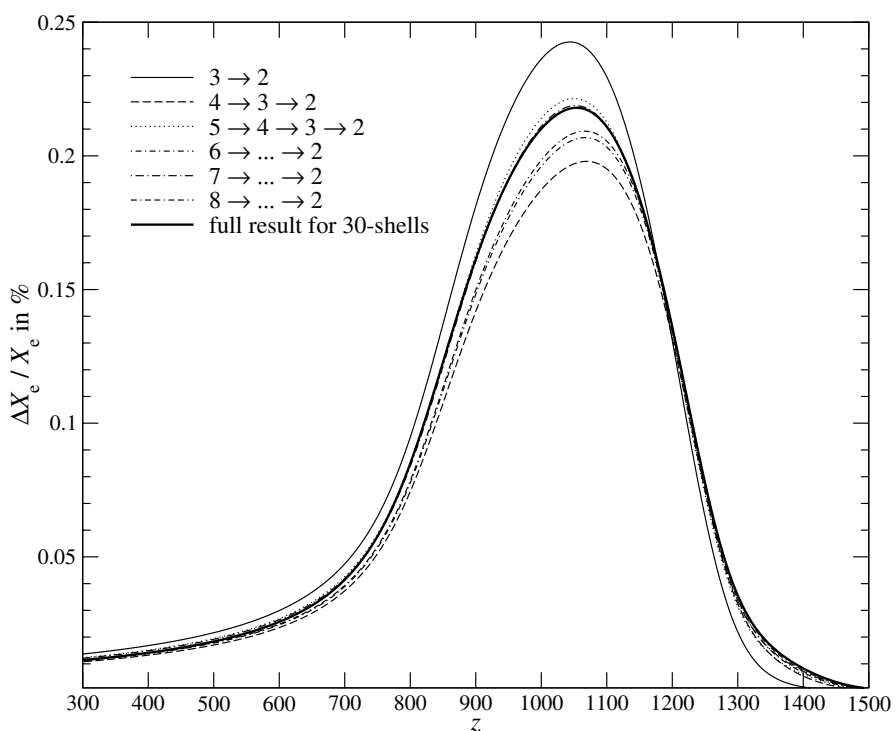

Fig. 2. Changes in the free electron fraction due to inclusion of Lymanseries feedback relative to the reference model without feedback. The computations were performed for a 15 -shell atom where for the Lymanseries the escape of photons was modeled using the Sobolev approximation. The curves are labeled according to the sequence of feedback that was included. For example, $4 \rightarrow 3 \rightarrow 2$ indicates that $\operatorname{Ly} \gamma \rightarrow \operatorname{Ly} \beta$ and $\operatorname{Ly} \beta \rightarrow \operatorname{Ly} \alpha$ feedback was taken into account. Also the full result for the 30-shell hydrogen atom is shown, where feedback up to $n=30$ was included.

emission in the Lyman- $\alpha$ line reduces by $\sim 0.8 \%$ at $z \sim 1232$. Note that this redshift is very close to the expected value, $z_{\mathrm{f}} \sim$ 1223, for the maximal feedback (see Table 1). On the other hand, due to the feedback-induced small changes in the populations of the levels, the net $2 \mathrm{~s}-1 \mathrm{~s}$ two-photon transition rate also increases with a maximum of roughly $\sim 0.5 \%$ at $z \sim 1034$ (see Fig. 4), which partially cancels the delaying feedback effect on the Lyman- $\alpha$ transition.

When including Lyman- $\gamma$ feedback, as expected, the emission in the Lyman- $\beta$ line reduces, with a maximal difference at the expected value $z \sim 1384$. Adding Lyman- $\delta$ feedback reduces the strength of the feedback of Lyman- $\gamma$ on the Lyman- $\beta$ transition by a significant fraction. Comparing the relative strength of the Lyman- $\delta$ at its maximum with the Lyman- $\gamma$ line at $z_{\mathrm{f}}$ (Fig. 1) yields $\Delta I_{v}^{\delta}\left(z_{\max }\right) / \Delta I_{v}^{\gamma}\left(z_{\mathrm{f}}\right) \sim 1 / 2$, which indicates that Lyman- $\delta$ should be able to affect Lyman- $\gamma$ strongly. Looking at the relative difference in the Lyman- $\gamma$ when including Lyman- $\delta$ feedback shows that at $z_{\mathrm{f}} \sim 1430$ the line is reduced by $\sim 50 \%$.

\section{The Lyman-continuum escape}

To estimate the maximal effect arising due to successful escape of photons from the Lyman-continuum we shall neglect any re-absorption of photons below the threshold frequency $v_{\mathrm{c}} \approx 3.288 \times 10^{15} \mathrm{~Hz}$. Although one can expect some modification due to the forest of lines in the Lyman-series close to the continuum $^{2}$, this approach will yield an upper limit on the effect, which turns out to be very small in any case.

${ }^{2}$ Within a small energy-distance $\xi$ below the ionization potential these in principle can be treated as a continuation of the photoionization cross-section (e.g. see Appendix II in Beigman et al. 1968).
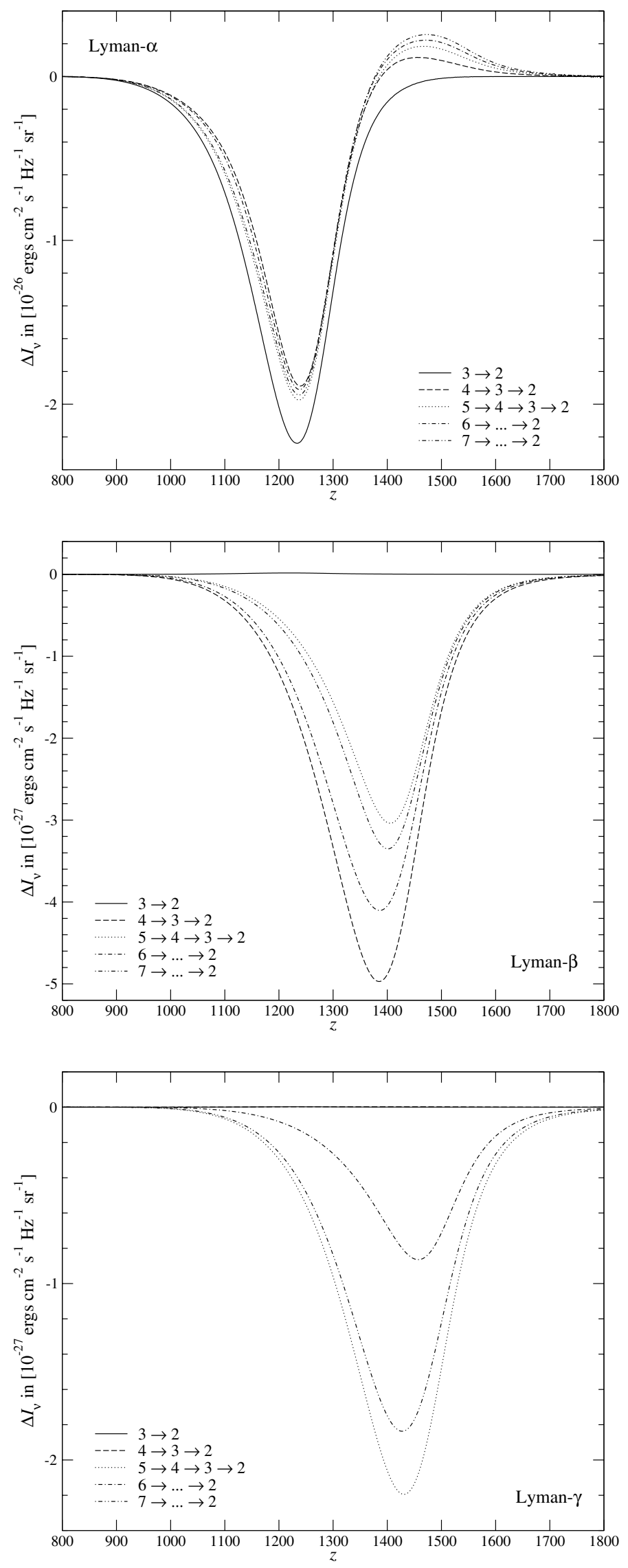

Fig. 3. Feedback-induced changes of $\Delta I_{v}\left(z_{\mathrm{em}}\right)$ for the Lyman- $\alpha$ and the modifications of the Lyman- $\beta$ and $\gamma$ lines before their absorption within the corresponding lower lying resonance. The results are based on the computations for a 15-shell hydrogen atom. 


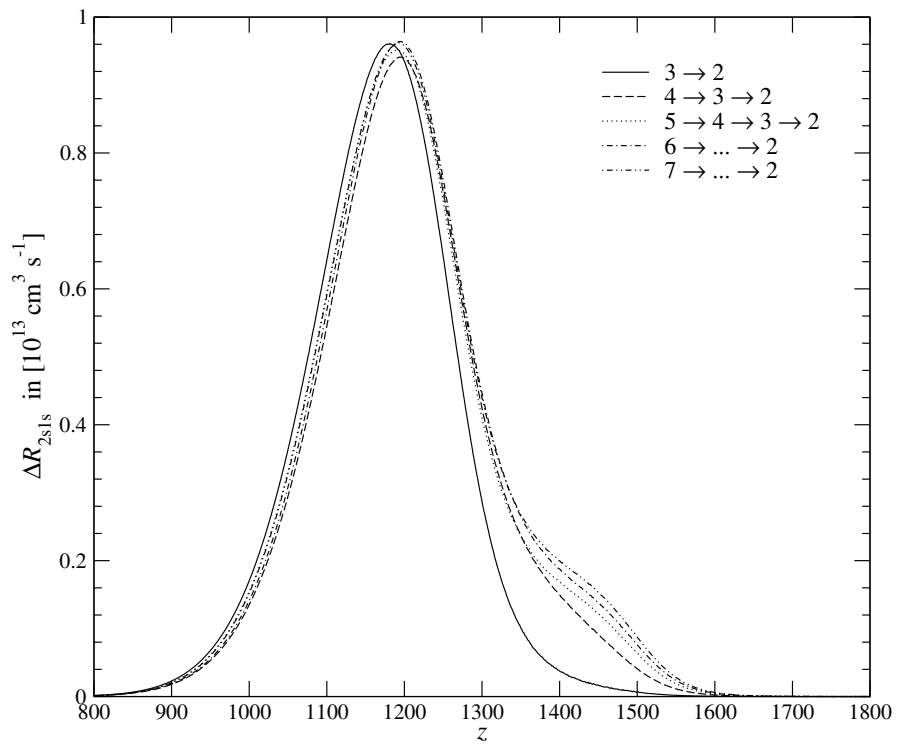

Fig. 4. Changes in the net 2s-1s two-photon decay rate due to the inclusion of $\operatorname{Ly} n \rightarrow \operatorname{Ly}(n-1)$ feedback, as labeled. The results are based on the computation for a 15-shell hydrogen atom.

With this in mind, in the vicinity of the Lyman-continuum the photon number only changes by recombination and ionization to the ground state (we neglect electron scattering):

$\left.\frac{1}{c} \frac{\partial N_{v}}{\partial t}\right|_{1 \mathrm{~s}} ^{\mathrm{rec}}=N_{\mathrm{e}} N_{\mathrm{p}} f\left(T_{\mathrm{e}}\right) \sigma_{1 \mathrm{sc}} \frac{2 v^{2}}{c^{2}} \mathrm{e}^{-\frac{h v}{k_{\mathrm{B}} T_{\mathrm{e}}}}-N_{1 \mathrm{~s}} \sigma_{1 \mathrm{sc}} N_{v}$.

Here $N_{\mathrm{e}}$ and $N_{\mathrm{p}}$ are the free electron and proton number densities, $N_{1 \mathrm{~s}}$ is the number density of hydrogen atoms in the ground state and $N_{v}=I_{v} / h v$, where $I_{v}$ is the photon intensity. In addition, $f\left(T_{\mathrm{e}}\right)=\left(N_{1 \mathrm{~s}} / N_{\mathrm{e}} N_{\mathrm{p}}\right)^{\mathrm{LTE}}$ and $\sigma_{1 \mathrm{sc}}$ is the $1 \mathrm{~s}$-photoionization cross-section. As can be seen from Eq. (1), photons are emitted with a spectrum ${ }^{3} \phi_{\mathrm{em}}^{\mathrm{rec}} \propto \sigma_{1 \mathrm{sc}}(v) v^{2} \mathrm{e}^{-h v / k_{\mathrm{B}} T_{\gamma}}$. We now compute the probability that one single recombination will lead to a successful escape of photons.

The optical depth for absorption of a photon, which has been emitted at redshift $z_{\mathrm{em}}$ and is observed at frequency $v_{\mathrm{obs}}$ at redshift $z_{\mathrm{obs}}$, within the Lyman-continuum is given by

$\tau_{\mathrm{c}}\left(z_{\mathrm{em}}, z_{\mathrm{obs}}, v_{\mathrm{obs}}\right)=c \int_{z_{\mathrm{obs}}}^{z_{\mathrm{em}}} \frac{N_{1 \mathrm{~s}} \sigma_{1 \mathrm{sc}}\left(v_{z}\right)}{H(1+z)} \mathrm{d} z$

where $v_{z}=v_{\text {obs }}(1+z) /\left(1+z_{\text {obs }}\right)$. If we substitute $\tilde{v}=v_{z}$ and use $\mathrm{d} \tilde{v}=v_{\text {obs }} \mathrm{d} z /\left(1+z_{\text {obs }}\right)$ then we have

$\tau_{\mathrm{c}}\left(z_{\mathrm{em}}, v_{\mathrm{em}}, v_{\mathrm{obs}}\right)=c \int_{v_{\mathrm{obs}}}^{v_{\mathrm{em}}} \frac{N_{1 \mathrm{~s}} \sigma_{1 \mathrm{sc}}(\tilde{v})}{H} \frac{\mathrm{d} \tilde{v}}{\tilde{v}}$.

Fixing the emission frequency and the emission redshift one can immediately determine the current redshift as a function of $\tilde{v}$ with $\tilde{v} / v_{\mathrm{em}}=(1+z) /\left(1+z_{\mathrm{em}}\right)$. Now the escape probability in the Lyman-continuum can be directly given in terms of the normalized emission profile $\phi_{\mathrm{em}}^{\mathrm{rec}}=\mathcal{N} \sigma_{1 \mathrm{sc}}(v) v^{2} \mathrm{e}^{-h v / k_{\mathrm{B}} T_{\gamma}}$ :

$P_{\mathrm{esc}}^{\mathrm{Ly}-\mathrm{c}}\left(z_{\mathrm{em}}\right)=\int_{v_{\mathrm{c}}}^{\infty} \phi_{\mathrm{em}}^{\mathrm{rec}}\left(v^{\prime}\right) \exp \left[-\tau_{\mathrm{c}}\left(z_{\mathrm{em}}, v^{\prime}, v_{\mathrm{c}}\right)\right] \mathrm{d} v^{\prime}$,

${ }^{3}$ Here and below we neglect the difference in the electron and photon temperature, which is smaller than $\Delta T / T \sim 10^{-6}-10^{-4}$ at most times during recombination.

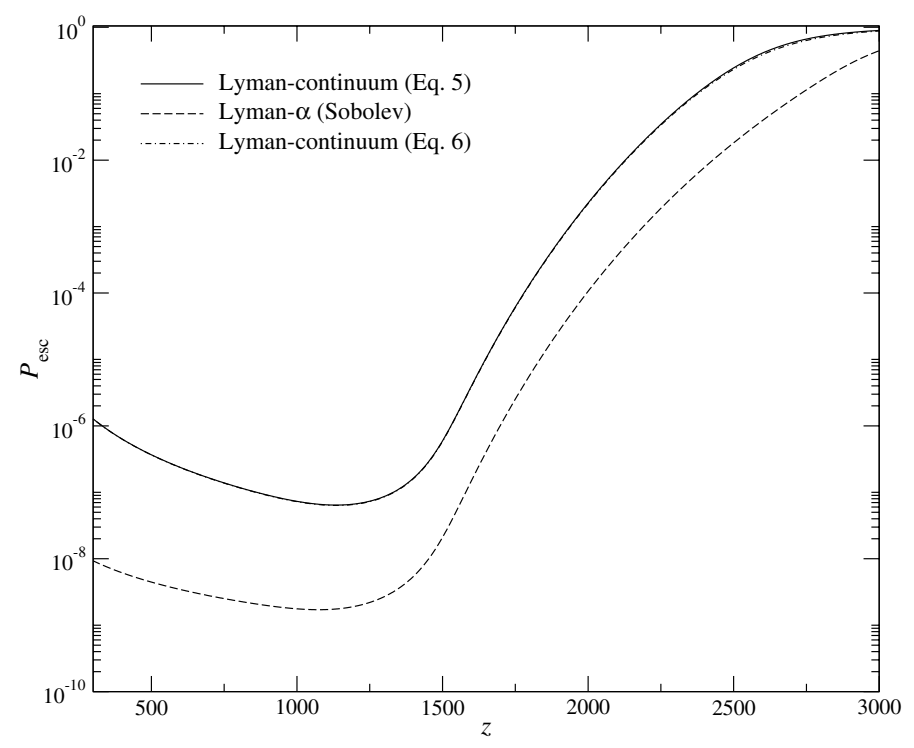

Fig. 5. Escape probability for the Lyman-continuum as given by Eq. (5) and Eq. (6). For comparison we also show the Sobolev escape probability for the Lyman- $\alpha$ photons.

where $\mathcal{N}$ is determined from the condition $\int \phi_{\mathrm{em}}^{\mathrm{rec}}(v) \mathrm{d} v=1$. Because $\phi_{\mathrm{em}}^{\mathrm{rec}}$ is sufficiently narrow $\left(\Delta v / v_{\mathrm{c}} \sim k_{\mathrm{B}} T_{\gamma} / h v_{\mathrm{c}} \sim 1 / 40\right.$ at $z \sim 1400)$ it only takes a very short time to cross the emission profile via redshifting. Since the characteristic time for changes of $N_{1 \mathrm{~s}}$ and $H$ is much longer, one can write

$P_{\mathrm{esc}}^{\mathrm{Ly}-\mathrm{c}}\left(z_{\mathrm{em}}\right) \approx \int_{v_{\mathrm{c}}}^{\infty} \phi_{\mathrm{em}}^{\mathrm{rec}}\left(v^{\prime}\right) \exp \left[-\tau_{0} \int_{v_{\mathrm{c}}}^{v^{\prime}} \frac{\hat{\sigma}_{1 \mathrm{sc}}(\tilde{v})}{\tilde{v}} \mathrm{~d} \tilde{v}\right] \mathrm{d} v^{\prime}$

where $\hat{\sigma}_{1 \mathrm{sc}}(v)=\sigma_{1 \mathrm{sc}}(v) / \sigma_{1 \mathrm{sc}}\left(v_{\mathrm{c}}\right)$ and the optical depth at the threshold frequency is $\tau_{0}\left(z_{\mathrm{em}}\right)=c \sigma_{1 \mathrm{sc}}\left(v_{\mathrm{c}}\right) N_{1 s}\left(z_{\mathrm{em}}\right) / H\left(z_{\mathrm{em}}\right)$.

It is possible to simplify Eq. (5) even further. First, since the emission profile is very narrow one may use $\phi_{\mathrm{em}}^{\mathrm{rec}} \approx$ $\tilde{\mathcal{N}} \mathrm{e}^{-h\left[v-v_{\mathrm{c}}\right] / k_{\mathrm{B}} T_{\gamma}}$, with the corresponding normalization constant $\tilde{\mathcal{N}}$. Assuming $\hat{\sigma}_{1 \mathrm{sc}} \approx\left[v_{\mathrm{c}} / v\right]^{3}$ one then obtains

$P_{\mathrm{esc}}^{\mathrm{Ly}-\mathrm{c}}\left(z_{\mathrm{em}}\right) \approx \int_{0}^{\infty} \mathrm{e}^{-\xi\left[1+\tau_{\mathrm{c}}^{\mathrm{esc}}\right]} \mathrm{d} \xi=\frac{1}{1+\tau_{\mathrm{c}}^{\mathrm{esc}}}$,

where $\tau_{\mathrm{c}}^{\mathrm{esc}}=\tau_{0} \frac{k_{\mathrm{B}} T_{\mathrm{e}}}{h v_{\mathrm{c}}}$. We see that interpreting the continuum profiles as a very narrow line ${ }^{4}, P_{\mathrm{esc}}^{\mathrm{Ly}-\mathrm{c}}$ appears to be similar to the Sobolev approximation for the escape of photons from resonance lines. In particular $P_{\mathrm{esc}}^{\mathrm{Ly}-\mathrm{c}}$ also scales as $1 / \tau_{\mathrm{c}}^{\mathrm{esc}}$ for large optical depth $\tau_{\mathrm{c}}^{\text {esc }} \gg 1$. However, unlike in the Sobolev approximation there is no symmetry between the absorption and emission profile, which actually is one of the key requirements that makes the Sobolev escape probability completely independent of the line profile. Comparing the results of Eqs. (5) and (6) shows that the latter equation provides a sufficient approximation to $P_{\mathrm{esc}}^{\mathrm{Ly}-\mathrm{c}}$ (see Fig. 5).

Using Eq. (6) it is easy to include the possibility of direct recombinations to the ground state connected with the escape of continuum photons. For this, one should add

$\Delta R_{\mathrm{c} 1 \mathrm{~s}} \approx P_{\mathrm{esc}}^{\mathrm{Ly}-\mathrm{c}}\left(z_{\mathrm{em}}\right) \times\left[N_{1 \mathrm{~s}} R_{1 \mathrm{sc}}-N_{\mathrm{e}} N_{\mathrm{p}} R_{\mathrm{c} 1 \mathrm{~s}}\right]$,

to the rate equations of the electrons and subtract this term for the $1 \mathrm{~s}$-equation. Here $R_{\mathrm{c} 1 \mathrm{~s}}$ and $R_{1 \mathrm{sc}}$ are the recombination and photoionization rate of the ground state, respectively. Note that we

\footnotetext{
${ }^{4}$ This is possible due to the exponential factor in $\phi_{\mathrm{em}}^{\mathrm{rec}}$.
} 


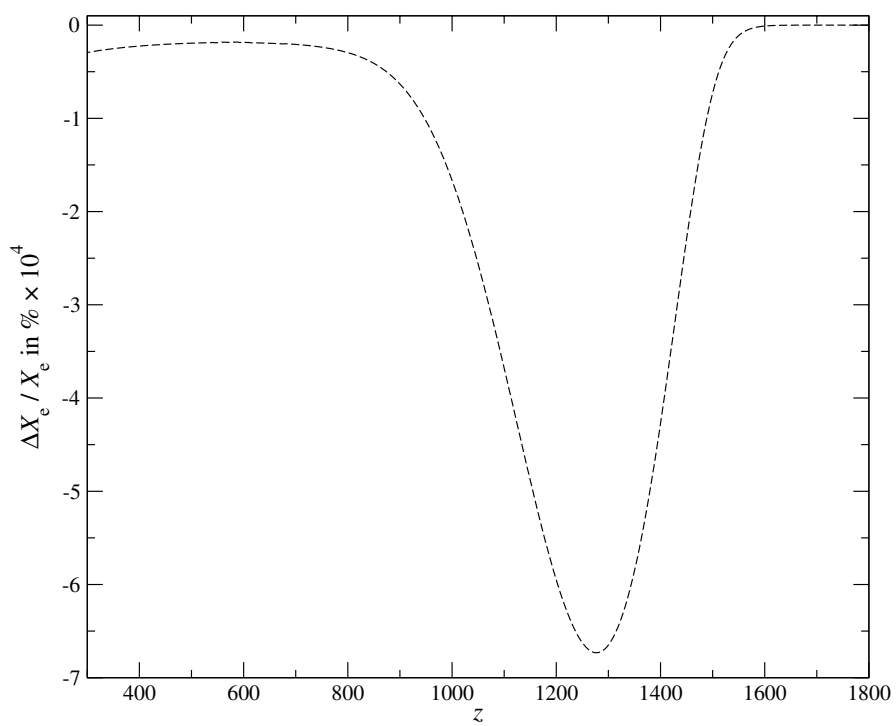

Fig. 6. Changes in the free electron fraction due to inclusion of Lymancontinuum escape relative to the reference model without any direct recombinations to $1 \mathrm{~s}$. The computations were performed for a 5-shell atom where for the Lyman-series escape the Sobolev approximation was used. Note that the absolute value of $\Delta X_{\mathrm{e}} / X_{\mathrm{e}}$ is $\sim 10^{-6}$ only.

assume that on the blue side of the Lyman-continuum the spectrum is Planckian. Therefore the photoionization rate $R_{1 \mathrm{sc}}$ does not include any spectral distortion. However, due to the release of photons during $\mathrm{He}$ I recombination some small distortions are created, which lead to an increase of the photoionization rate.

Comparing the escape probability for the Lyman-continuum with the Sobolev escape probability for the Lyman- $\alpha$ photons (see Fig. 5) one can see that $P_{\mathrm{esc}}^{\mathrm{Ly}-\mathrm{c}}\left(z_{\mathrm{em}}\right)$ is roughly 10-100 times larger at most times. However, including the possibility of direct recombinations to the ground state in our recombination code yields a tiny correction to $X_{\mathrm{e}}$ (see Fig. 6), which shows that $\Delta R_{\mathrm{c} 1 \mathrm{~s}}$ as given by Eq. (7) is still many orders of magnitude smaller than the net Lyman- $\alpha$ transition rate. One can safely neglect direct recombinations to the ground state of hydrogen for computations of the recombination history.

\section{Discussion}

We have shown that $\mathrm{Ly} n \rightarrow \operatorname{Ly}(n-1)$ feedback leads to a modification in the ionization history of $\Delta N_{\mathrm{e}} / N_{\mathrm{e}} \sim 0.22 \%$ at $z \sim 1050$. Since this is very close to the peak of the Thomson-visibility function (Sunyaev \& Zeldovich 1970), corrections to the CMB power spectra of similar order are expected. Computing the CMB $T T$ and $E E$ power spectra using the results for the ionization history with and without the additional feedback we find changes with a typical peak to peak amplitude $\left|\Delta C_{l}^{T T} / C_{l}^{T T}\right| \sim$ $0.15 \%$ and $\left|\Delta C_{l}^{E E} / C_{l}^{E E}\right| \sim 0.36 \%$ at $l \lesssim 3000$ (see Fig. 7).

As shown by Chluba \& Sunyaev (2007) due to two-photon processes one can expect some asymmetries in the emission profiles of the higher Lyman-series lines. This could modify the amount and time-dependence for the $\operatorname{Ly} n \rightarrow \operatorname{Ly}(n-1)$ feedback process and may lead to additional differences in the results presented above. However, because the bulk of photons that escape in the red wing of a particular Lyman-transition is expected to come from close to the line center, this may be of minor importance. Here the most interesting aspect may be the changes in the time-dependence of the feedback, but more detailed computations would be necessary to understand this problem.

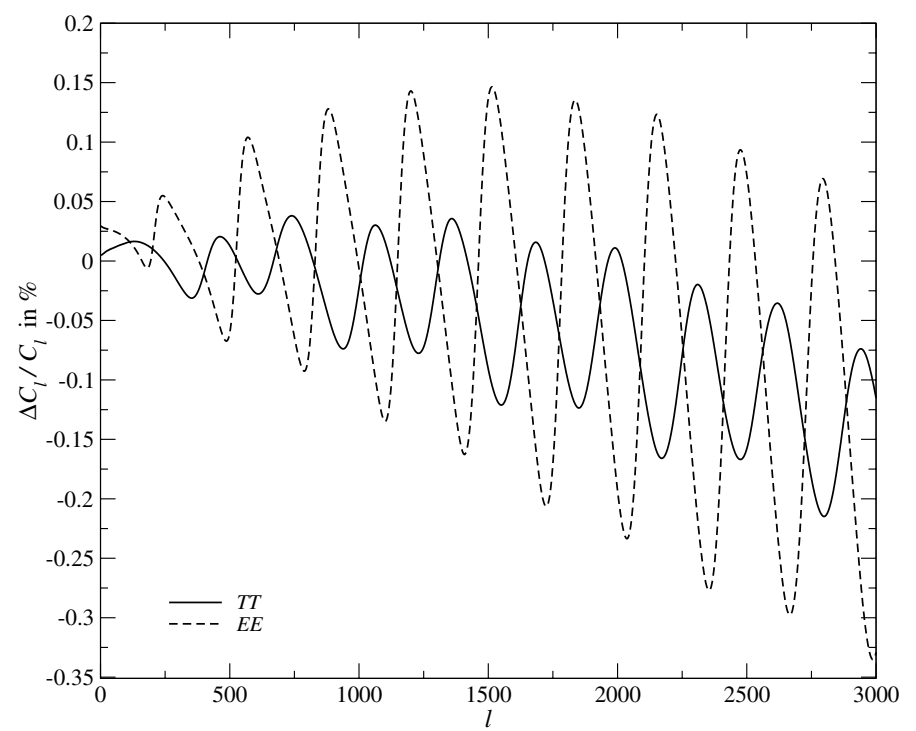

Fig. 7. Changes in the CMB temperature $(T T)$ and polarization $(E E)$ power spectra. The differences were computed using our modified versions of CMBEASY (Doran 2005), which allows loading of pre-calculated recombination histories, and where the corresponding RECFAST-routine was improved to achieve higher numerical accuracy with solvers from the NAG-library.

In addition one should look at the feedback-induced corrections in the hydrogen recombination history due to the He I spectral distortions, in particular due to the photons appearing in the $2^{1} \mathrm{P}_{1} \rightarrow{ }^{1} \mathrm{~S}_{0}$ resonance transition, the $2^{3} \mathrm{P}_{1} \rightarrow{ }^{1} \mathrm{~S}_{0}$ intercombination line and the $2^{1} \mathrm{~S}_{0} \rightarrow 1^{1} \mathrm{~S}_{0}$ two-photon continuum. It is clear that all high frequency He II lines will be reprocessed during He I recombination, and correspondingly can only affect the ionization history during that epoch. However, for the feedback of the He I lines on hydrogen recombination it will be important to compute the re-processing of photons in the Lyman-continuum and all the subsequent Lyman-series transitions. Since all of these transitions are very optically thick a huge part of the photons released during $\mathrm{He}$ I and $\mathrm{He}$ II recombination will never reach the observer today, but due to re-absorption by neutral hydrogen, they are fully converted to hydrogen Lyman$\alpha$ and 2 s-1s continuum photons (see also Kholupenko et al. 2007; Rubiño-Martín et al. in preparation). Since most of the helium $2{ }^{1} \mathrm{P}_{1} \rightarrow{ }^{1} \mathrm{~S}_{0}$ photons are released at $z \sim 1800-2600$, they should be re-absorbed by neutral hydrogen atoms at the early stages $z \gtrsim 1200-1400$. One can also conclude this from the paper of Kholupenko et al. (2007), where the re-processed $\mathrm{He}$ II $\rightarrow$ He I photons appear on the red side of the hydrogen Lyman- $\alpha$ distortion (most hydrogen Lyman- $\alpha$ photons are released at $z \sim 1400$ ). Therefore the helium photons affect the hydrogen recombination history well before the maximum of the Thomson-visibility function $(z \sim 1100)$, and hence should have a rather small impact on the CMB power spectra. However, a more careful computation is required and will be described in a forthcoming paper (Rubiño-Martín et al. in preparation).

Acknowledgements. The authors thank the anonymous referee for his useful comments.

\section{References}

Beigman, I. L., \& Sunyaev, R. A. 1978, preprint Leb. Phys. Inst. N163 Beigman, I. L., Vainshtein, L. A., \& Syunyaev, R. A. 1968, Soviet Phys. Uspekhi, 11,411 
Burgin, M. S. 2003, Astron. Rep., 47, 709

Chluba, J., \& Sunyaev, R. A. 2006a, A\&A, 458, L29

Chluba, J., \& Sunyaev, R. A. 2006b, A\&A, 446, 39

Chluba, J., \& Sunyaev, R. A. 2007, ArXiv e-prints, 705

Chluba, J., Rubiño-Martín, J. A., \& Sunyaev, R. A. 2007, MNRAS, 374, 1310

Doran, M. 2005, J. Cosmol. Astro-Part. Phys., 10, 11

Dubrovich, V. K. 1975, Sov. Astron. Lett., 1, 196

Dubrovich, V. K., \& Stolyarov, V. A. 1995, A\&A, 302, 635

Dubrovich, V. K., \& Shakhvorostova, N. N. 2004, Astron. Lett., 30, 509

Dubrovich, V. K., \& Grachev, S. I. 2005, Astron. Lett., 31, 359

Hinshaw, G., Nolta, M. R., Bennett, C. L., et al. 2006, ArXiv Astrophysics e-prints

Kholupenko, E. E., \& Ivanchik, A. V. 2006, Astron. Lett., 32, 795

Kholupenko, E. E., Ivanchik, A. V., \& Varshalovich, D. A. 2005, Gravit. Cosmol., 11, 161

Kholupenko, E. E., Ivanchik, A. V., \& Varshalovich, D. A. 2007, MNRAS, L42
Lewis, A., Weller, J., \& Battye, R. 2006, MNRAS, 373, 561

Page, L., Hinshaw, G., Komatsu, E., et al. 2006, ArXiv Astrophysics e-prints Peebles, P. J. E. 1968, ApJ, 153, 1

Rubiño-Martín, J. A., Chluba, J., \& Sunyaev, R. A. 2006, MNRAS, 371, 1939

Rubiño-Martín, J. A., Chluba, J., \& Sunyaev, R. A. 2007, in preparation

Rybicki, G. B. \& dell'Antonio, I. P. 1993, in Observational Cosmology, ed. G. L.

Chincarini, A. Iovino, T. Maccacaro, \& D. Maccagni, ASP Conf. Ser. 51, 548 Seager, S., Sasselov, D. D., \& Scott, D. 1999, ApJ, 523, L1

Seager, S., Sasselov, D. D., \& Scott, D. 2000, ApJS, 128, 407

Sunyaev, R. A. \& Zeldovich, Y. B. 1970, Astrophysics and Space Science, 7, 3 Switzer, E. R. \& Hirata, C. M. 2007, ArXiv Astrophysics e-prints

Wong, W. Y. \& Scott, D. 2007, MNRAS, 375, 1441

Wong, W. Y., Seager, S., \& Scott, D. 2006, MNRAS, 367, 1666

Zeldovich, Y. B., Kurt, V. G., \& Syunyaev, R. A. 1968, Zhurnal Eksperimental noi i Teoreticheskoi Fiziki, 55, 278 